\title{
The relationship between air pollution and asthma in Malaysian schoolchildren
}

\begin{abstract}
Rapid increases in traffic volumes in countries such as Malaysia may lead to exposure to poor air quality both outdoors and indoors. This study investigated asthma symptoms among Malaysian schoolchildren in relation to indoor and outdoor air pollution in urban and semirural settings in a school-based cross-sectional study of 1,952 schoolchildren living in urban and semi-rural areas. Parents completed a questionnaire including questions on asthma symptoms and environmental risk factors. Air pollutant concentrations were measured at 16 schools. Analysis used multiple logistic regression controlling for sociodemographic and indoor air pollution factors. A total of 11.4, 7.6, 9.5 and 7.0 \% schoolchildren had asthma, current wheeze, dry cough at night and wheezing after exercise, respectively. Asthma and dry cough were significantly higher among urban schoolchildren. A $10-\varepsilon g / \mathrm{m} 3$ increase in outdoor NO2 level was associated with a twofold increase in the reporting of current wheeze (odds ratio (OR) 1.90; $95 \%$ confidence interval (CI) 1.02ï 3.52); a 10-عg/m3 increase in indoor PM2.5 was similarly associated with a twofold increase in the reporting of wheeze during exercise (OR 2.08; 95 \% CI 1.02ï 4.26). Secondhand smoke (SHS), mold and the presence of new furniture at home were also linked to asthma symptoms. NO2 concentrations in urban Malaysia frequently exceed those of the international standards. Exposure to trafficrelated air pollution may be linked to asthma symptoms among children in Malaysia.
\end{abstract}

Keyword: Indoor air quality; Urban; Semi-rural; Traffic-related pollution; Asthma; Children 